Pacific

Journal of

Mathematics

\title{
ON A THEOREM OF LANCASTER AND SIEGEL
}

Danzhu Shi and Robert Finn 


\section{ON A THEOREM OF LANCASTER AND SIEGEL}

\section{DANZHu Shi And Robert Finn}

In establishing conditions for continuity of the height of a capillary surface $f(x, y)$ at a re-entrant corner point of the domain of definition, Lancaster and Siegel introduced a hypothesis of symmetry, which does not appear in corresponding conditions for a protruding corner. We show here that the hypothesis cannot be discarded. Starting with a symmetric configuration for which the surface height is continuous at the corner point in accordance with the hypotheses of those authors, we show that the height can be made discontinuous by an asymmetric domain perturbation that is in an asymptotic sense arbitrarily small, and for which all hypotheses other than that of symmetry remain in force.

In a downward directed gravity field, the height $f(x, y)$ of a capillary surface interface is determined, up to an additive constant depending on an eventual volume constraint, as a solution of

$$
\nabla \cdot T f=\kappa f, \quad T f \equiv \frac{\nabla f}{\sqrt{1+|\nabla f|^{2}}}
$$

corresponding to a (physical) constant $\kappa>0$. On the boundary $\partial \Omega$ one finds the condition

$$
\nu \cdot T u=\cos \gamma
$$

where $\nu$ is unit exterior normal, and $\gamma$ the (prescribed) contact angle between the solution surface and the bounding cylinder walls over $\partial \Omega$. We may assume $0 \leq \gamma \leq \pi$. The unique existence of solutions of (1) and (2) in a piecewise smooth bounded domain $\Omega$ follows from the material in [2], [4], $[7]$ and [8]. It should be noted that Condition (2) is imposed only on the smooth portions of the boundary; no condition is required at corner points, nor is there any a-priori growth restriction at those points.

Lancaster and Siegel [6] have characterized remarkable properties of radial limits $R f$ of solutions $f(x, y)$ at eventual corner points $O \in \partial \Omega$. The limit exists for every direction of approach at the corner points, but its dependence on direction can vary considerably, depending on circumstances. By Corollary 1 of [6], if $\partial \Omega$ is of class $C^{(1)}$ except at the corner points, then the solutions are continuous up to all boundary points with the possible exception of corner points. 
For a protruding corner of opening $2 \alpha<\pi$, Corollary 4 of [6] yields that for all $\gamma$ in the range $\pi / 2-\alpha<\gamma<\pi / 2+\alpha, R f$ is finite and is independent of direction, and thus $f$ is continuous at the corner. If $\gamma$ lies exterior to this range, it was shown by Concus and Finn [1] that no bounded solution exists.

For a re-entrant corner of opening $2 \alpha>\pi$, the situation is more complicated. It follows from Theorem 5.2 of [3] that all solutions are bounded at such a point; however Korevaar showed [5] that solutions can be discontinuous at re-entrant corner points. Lancaster and Siegel proved in [6]:

Corollary 2. Suppose $\pi / 2 \leq \alpha<\pi$ and that $\Omega$ is symmetric under reflection in the $x$-axis, with the $x$-axis bisecting the corner point. Suppose further that either

$$
\alpha-\pi / 2 \leq \gamma \leq \pi / 2 \quad \text { or } \pi / 2 \leq \gamma \leq 3 \pi / 2-\alpha .
$$

Then the (uniquely determined) solution $f(x, y)$ of (1) and (2) in $\Omega$ is symmetric under reflection in the $x$-axis, and continuous at $O$.

Condition (3) is analogous to the condition of Corollary 4 just mentioned for a protruding corner. The authors show that also in this case their result is sharp with respect to that condition, by providing a counterexample under the given symmetry when $\gamma$ is outside the specified range. However symmetry was not needed for Corollary 4 , and the question arises, as to the extent it is essential for Corollary 2. This question is not addressed in [6].

In the present note, we show that in general (3) does not suffice for continuity, and the symmetry hypothesis cannot be significantly relaxed. In fact, in an asymptotic sense to be defined, there exist configurations that deviate arbitrarily little from symmetry, for which (3) holds and for which the solution $f(x, y)$ fails to be continuous at $O$.

We construct an example of such behavior as a modification of the counterexample just indicated. We assume $\pi / 2<\alpha<\pi$, and consider the case $\gamma=\alpha-\pi / 2$, which satisfies (3). As starting point we repeat some steps in the reasoning of $[\mathbf{6}]$.

Figure 1 illustrates a particular configuration. This is basically Figure 10 in [6], the only change significant for us being that two vertical boundary lines in that figure are now introduced with finite slopes that are negatives of each other.

As in [6], we introduce the disk $\mathcal{D} \subset \Omega$ of radius $R_{0}$, tangent to the $y$-axis at $O$. By Theorem 5.2 of [5], any solution $f(x, y)$ of $(1)$ in $\mathcal{D}$ satisfies

$$
\sup _{\mathcal{D}} f<\frac{2}{\kappa R_{0}}+R_{0}
$$

throughout $\mathcal{D}$ (one needs a slightly strengthened version of the theorem, which is routine to obtain). The choice $R_{0}=\sqrt{2 / \kappa}$ yields the best possible 
bound from (4); we restrict attention to domains $\Omega$ that contain this disk, and we are led to

$$
\sup _{\mathcal{D}} f<2 \sqrt{\frac{2}{\kappa}}
$$

in $\mathcal{D}$. Thus, (5) holds also for the radial limit $R f$ along any line segment within $\mathcal{D}$ leading to $O$.

We denote by $\varepsilon$ the distance between the lines $L$ and $L^{*}$ of Figure 1 . We choose $\gamma^{*}$ in the range $\gamma \leq \gamma^{*}<\pi-\gamma$ and introduce the two symmetrically placed circular $\operatorname{arcs} \mathcal{C}_{1}, \mathcal{C}_{2}$ of equal radii $r$, determined by

$$
\varepsilon=r\left(\cos \gamma+\cos \gamma^{*}\right)
$$

and meeting the line $L$ in angle $\gamma$. These arcs meet $L^{*}$ in the angle $\gamma^{*}$. We construct a torus $\mathcal{T}$ of sectional radius $r$ which contains $\mathcal{C}_{1}, \mathcal{C}_{2}$, and we denote by $2 R$ the distance between the centers of $\mathcal{C}_{1}, \mathcal{C}_{2}$. Consider the portion of $\mathcal{T}$ that lies above the plane of the arcs, and choose the underside $g(x, y)$ of that surface as a "comparison surface". This surface has the general appearance of a footbridge in a Japanese garden. Note that it is vertical on the circles $\mathcal{C}_{1}, \mathcal{C}_{2}$, and that it meets vertical walls through $L, L^{*}$ in the constant angles $\gamma, \gamma^{*}$. The surface $g(x, y)$ has at each of its points a mean curvature

$$
H_{g} \geq \frac{1}{2}\left(\frac{1}{r}-\frac{1}{R-r}\right)
$$

and $\cos \gamma^{*} \leq \cos \gamma$. Theorem 5.1 of [ 5$]$ now yields that in the domain $\mathcal{D}_{\mathcal{T}} \subset \Omega$ onto which $\mathcal{T}$ projects, there holds

$$
\inf _{\mathcal{D}_{\mathcal{T}}} f>\frac{1}{\kappa}\left(\frac{1}{r}-\frac{1}{R-r}\right)-R .
$$

We now change the perspective somewhat. Instead of starting with the channel width $\varepsilon$ and angle $\gamma^{*}$ as above, we choose $r=r_{0}$ so that

$$
\frac{1}{\kappa}\left(\frac{1}{r_{0}}-\frac{1}{R-r_{0}}\right)-R>2 \sqrt{\frac{2}{\kappa}} .
$$

We retain this choice for $r$ throughout the ensuing discussion. The choice imposes a relation between $\varepsilon$ and $\gamma^{*}$, according to (6), which is formally satisfied, for any $\varepsilon$ in the range $0<\varepsilon \leq r_{0}(1+\cos \gamma)$, by a unique value $\gamma^{*}$ for which $0 \leq \gamma^{*}<\pi-\gamma$. We observe that any $r_{0}$ chosen as above satisfies $r_{0}<R_{0}$.

We would like to position $\mathcal{C}_{2}$ so that the intersection with $L$ will be at $O$. From the relations (5), (8) and (9) we could then conclude that $f(x, y)$ is discontinuous at $O$. In the configuration of Figure 1 that cannot be done, as the other endpoint of $\mathcal{C}_{2}$ would then no longer meet $L^{*}$, and Theorem 5.1 of [5] could no longer be applied. That is of course consistent with the 
original result of [2], that in the symmetric configuration the solution must be continuous at $O$.

By abandoning symmetry, we can however modify the configuration to permit the construction. We tilt the $y$-axis clockwise through an angle $\delta$ to be determined, so that it becomes an inclined line $L^{\delta}$, and we shift the disk $\mathcal{D}$ so that it is tangent to $L^{\delta}$ at $O$. We then extend $L^{*}$ until it meets $\partial \mathcal{D}$ at a point $p(\delta)$, and we shorten $L^{* *}$ so that it does not extend beyond $L^{\delta}$. Finally, we choose $\delta$ so that the two circles $\mathcal{C}_{2}$ and $\partial \mathcal{D}$ meet at that same point $p(\delta)$, when $\mathcal{C}_{2}$ meets $L$ at $O$. If $\delta$ is small enough, $\mathcal{D}$ will continue to lie within $\Omega$. We will show that such a choice is always available, corresponding to the value of $r=r_{0}$ chosen above, and with $\gamma^{*}$ within the required range, if $\varepsilon$ is sufficiently small. For the moment we postpone verification of this requirement.

Local details of the construction are indicated in Figures 2 and 3. In this configuration, $\mathcal{T}$ can be positioned to contain the $\operatorname{arc} \mathcal{C}_{2}$, and a segment extending to $O$ can be found within $\mathcal{D}_{\mathcal{T}}$. On such a segment, the lower bound (8) holds. However, on any segment extending to $O$ within $\mathcal{D}$ we have the upper bound (5). In view of (9), the asserted discontinuity of $f$ at $O$ follows. But all conditions for Corollary 2 are fulfilled except for symmetry. Subject to verification that $\delta$ is determined by the requirements and tends to zero with $\varepsilon$, we find that the symmetry hypothesis of the corollary is necessary for continuity at $O$.

The formal analytical criterion for determining $\delta(\varepsilon)$ is contained in the equations

$$
\begin{aligned}
\cos \gamma+\cos \gamma^{*} & =\varepsilon / r_{0} \\
\cos (\gamma+\delta)+\cos \left(\gamma^{*}+\delta\right) & =\varepsilon / R_{0} .
\end{aligned}
$$

Here (10a) is a repetition of (6); it follows immediately from the construction of $\mathcal{C}_{1}, \mathcal{C}_{2}$. We obtain (10b) similarly using the placement of $\mathcal{D}$ and additionally that $\mathcal{C}_{2}$ and $\partial \mathcal{D}$ intersect at $O$ and at $p$ in equal angles; see Figure 3 for the configuration.

Keeping $r_{0}$ fixed, we let $\varepsilon \rightarrow 0$, and observe that $\gamma^{*}$, as determined from (10a), then increases toward $\pi-\gamma$. Specifically, from (10a) a smooth function branch

$$
\gamma^{*}=\varphi(\varepsilon) \equiv \arccos \left(\frac{\varepsilon}{r_{0}}-\cos \gamma\right)
$$

is determined, for which $\varphi(0)=\pi-\gamma$. For this branch, we find

$$
\varphi^{\prime}(\varepsilon)=-1 / r_{0} \sin \varphi(\varepsilon)
$$

and thus $\varphi^{\prime}(0)=-1 / r_{0} \sin \gamma$. We conclude $\gamma^{*}<\pi-\gamma$, and $\gamma^{*} \geq \gamma$ if $\varepsilon$ is small enough. We place the function (11) into (10b), and solve the resulting 
implicit relation for a branch $\delta(\varepsilon)$, with

$$
\delta(\varepsilon)=\arccos \left(\frac{\varepsilon}{2 R_{0} \cos \frac{\gamma-\gamma^{*}}{2}}\right)-\frac{\gamma+\gamma^{*}}{2},
$$

such that $\delta(0)=0$. Since $\varphi(0)-\gamma=\pi-2 \gamma$ and $0<\pi-2 \gamma<\pi$, this procedure determines the required $\delta(\varepsilon)$ explicitly.

For the distance $d(\varepsilon)$ to which $L^{*}$ must be extended to meet $L^{\delta}$, we obtain after some manipulation, using Figure 3,

$$
d(\varepsilon)=\frac{\varepsilon}{\sin \gamma} \frac{\cos \left(\frac{\gamma+\gamma^{*}}{2}\right)}{\cos \left(\frac{\gamma-\gamma^{*}}{2}\right)} .
$$

The denominator in (14) is harmless, as just observed. For the numerator, we have

$$
\begin{aligned}
\cos \frac{\gamma+\gamma^{*}}{2} & =\left[\frac{1+\cos \left(\gamma+\gamma^{*}\right)}{2}\right]^{\frac{1}{2}} \\
& =\frac{\sqrt{2}}{2}\left[1+\cos \gamma \cos \gamma^{*}-\sin \gamma \sin \gamma^{*}\right]^{\frac{1}{2}}
\end{aligned}
$$

In view of $(10 \mathrm{a})$ we obtain

$$
\begin{aligned}
& \cos \frac{\gamma+\gamma^{*}}{2} \\
& =\frac{\sqrt{2} \sin \gamma}{2}\left[1+\frac{\varepsilon \cos \gamma}{r_{0} \sin ^{2} \gamma}-\left(1+\frac{2 \varepsilon \cos \gamma}{r_{0} \sin ^{2} \gamma}-\frac{\varepsilon^{2}}{r_{0}^{2} \sin ^{2} \gamma}\right)^{\frac{1}{2}}\right]^{\frac{1}{2}} .
\end{aligned}
$$

From the inequality $\sqrt{1+x}>1+(x / 2)-\left(x^{2} / 8\right), x>0$, applied to the inner root, we are led to

$$
\cos \frac{\gamma+\gamma^{*}}{2}<C \varepsilon
$$

for a fixed constant $C$, as $\varepsilon \rightarrow 0$, and hence $d(\varepsilon)=\mathcal{O}\left(\varepsilon^{2}\right)$, from (14). A similar estimate applies to the amount $d^{*}$ by which $L^{*}$ must be shortened. Thus $d$ and $d^{*}$ decrease faster than $\varepsilon$. Under a coordinate normalization holding the width of the strips constant as $\varepsilon \rightarrow 0$, the new distance $\widetilde{d}(\varepsilon)$ retains the order $\widetilde{d}(\varepsilon)=\mathcal{O}(\varepsilon)$. It is in that asymptotic sense that there exist configurations that deviate arbitrarily little from symmetry, for which the solution $f(x, y)$ fails to be continuous at $O$, as asserted above. This behavior is also evident geometrically, directly from the nature of the construction.

Acknowledgements. This investigation was supported in part by the National Science Foundation. The authors are indebted to the Max-PlanckInstitut für Mathematik in den Naturwissenschaften, in Leipzig, for its hospitality during the course of the study. We wish to thank David Siegel for helpful comments that have led to improvements in our exposition. 


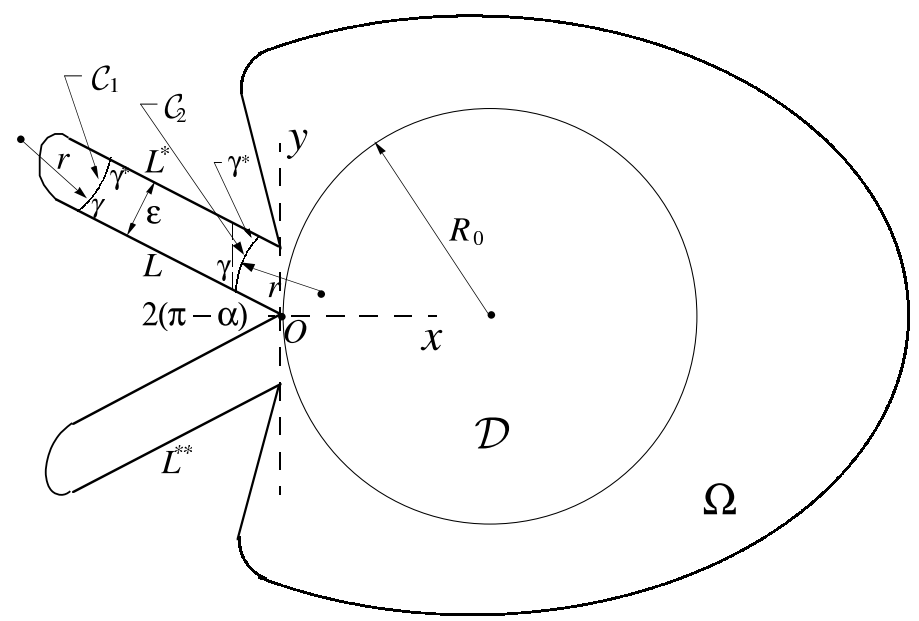

Figure 1. Domain $\Omega$ leading to continuity at $O$ of capillary surface with contact angle $\gamma=\alpha-\pi / 2$.

Figure 1. Domain $\Omega$ leading to continuity at $O$ of capillary surface with contact angle $\gamma=\alpha-\pi / 2$. 


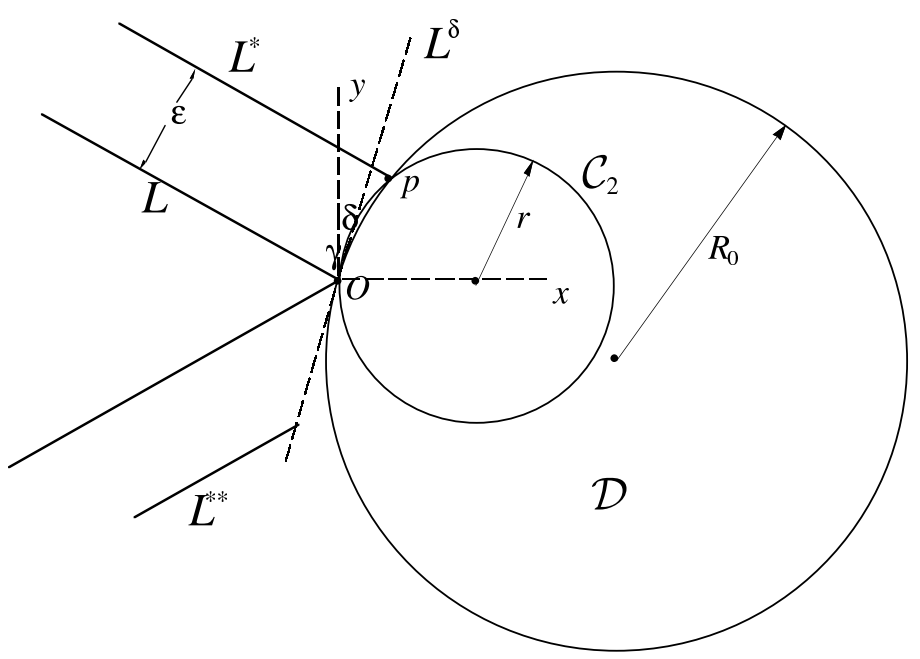

Figure 2. Details of construction for example of discontinuity at $O$. The two circles are tangent respectively to the vertical and to $L^{\delta}$ at $O$, and both pass through $p$.

Figure 2. Details of construction for example of discontinuity at $O$. The two circles are tangent respectively to the vertical and to $L^{\delta}$ at $O$, and both pass through $p$.

\section{References}

[1] P. Concus and R. Finn, On capillary free surfaces in a gravitational field, Acta Math., 132 (1974), 207-223, MR 58 \#32327c, Zbl 0382.76005. 


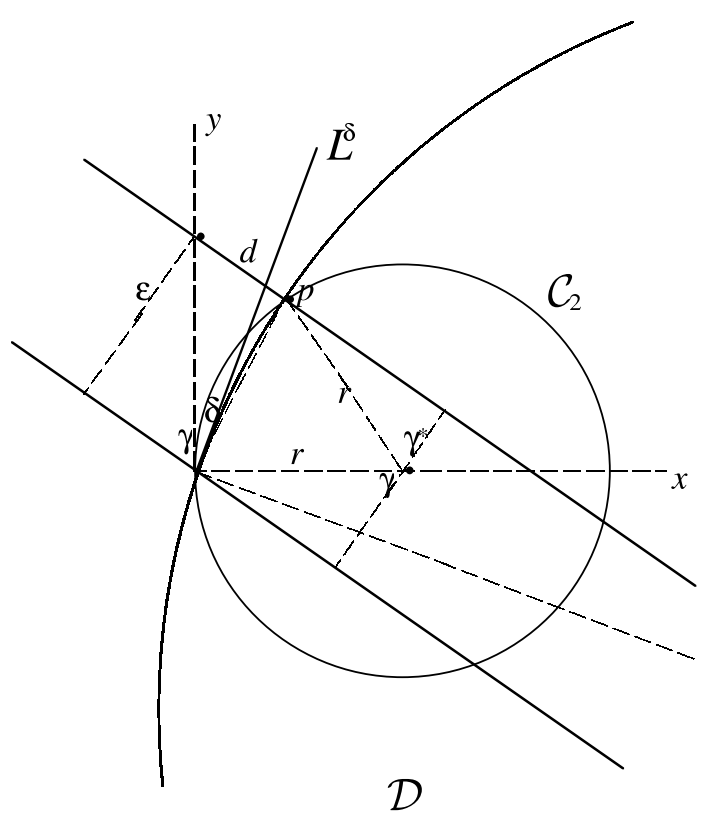

Figure 3. Further details of the construction.

Figure 3. Further details of the construction.

[2] M. Emmer, Esistenza, unicità e regolarità nelle superfici di equilibrio nei capillari, Ann. Univ. Ferrara Sez. VII (N.S.), 18 (1973), 79-94, MR 49 \#1281, Zbl 0275.49005.

[3] R. Finn, Equilibrium Capillary Surfaces, Springer-Verlag, New York, 1986; Russian translation (with Appendix by H.C. Wente), Mir Publishers, 1988, MR 88f:49001, Zbl 0583.35002 . 
[4] R. Finn and C. Gerhardt, The internal sphere condition and the capillary problem, Ann. Mat. Pura Appl. (4), 112 (1997), 13-31, MR 55 \#7106, Zbl 0349.49019.

[5] N.J. Korevaar, On the behavior of a capillary surface at a re-entrant corner, Pacific J. Math., 88(2) (1980), 379-385, MR 82c:49033, Zbl 0475.49034.

[6] K. Lancaster and D. Siegel, Existence and behavior of the radial limits of a bounded capillary surface at a corner, Pacific J. Math., 176 (1996), 165-194; Editorial corrections in Pacific J. Math., 179 (1997), 397-402, MR 98g:58030a, MR 98g:58030b, Zbl 0866.76018.

[7] N.N. Ural'tseva, Solvability of the capillary problem, Vestnik Leningrad. Univ. Mat., (1973) No. 19, 54-64; English translation in Vestn. Leningr. Univ. Math., 6 (1979), 363-375, Zbl 0419.35040.

[8] _ Solvability of the capillary problem II, Vestnik Leningrad. Univ. Mat., (1975) No. 1, 143-149; English translation in Vestn. Leningr. Univ. Math., 8 (1980), 151-158, Zbl 0436.35023.

Received September 8, 2002 and revised February 14, 2003.

Mathematics Department

STANFORD UNIVERSITY

StANFORD, CA 94305-2125

E-mail address: sdanzhu@stanford.edu

Mathematics Department

STANFORD UNIVERSITY

STANFORD, CA 94305-2125

E-mail address: finn@math.stanford.edu 